\title{
LA ULTRASONOGRAFÍA EN BOVINOS
}

\section{ULTRASONOGRAPHY OF THE BOVINE}

\author{
Diana E. Gutiérrez-Lizarazoํㅜ, Giovanni M. Báez-Sandoval²
}

\begin{abstract}
Forma de citar: GUTIÉRREZ-LIZARAZO Diana, BÁEZ-SANDOVAL Giovanni. La ultrasonografía en bovinos.
\end{abstract} Respuestas. 2014; 19(1):99-106.

Recibido:

Septiembre 9 de 2013

Aceptado:

Diciembre 10 de 2013

${ }^{1}$ Ingeniera Pecuaria UFPS. DG Reproducción Animal. dianaegutierrezl@dgrepro.

com

${ }^{2} \mathrm{PhD}$ Candidate. Department of Dairy Science, University of Wisconsin. Madison, WI. 53706

baezsandoval@wisc.edu Universidad Francisco de Paula Santander.

\section{RESUMEN}

Antecedentes: La ultrasonografía transrectal se ha convertido en una útil herramienta en la reproducción animal desde su implementación en la década de 1980. Su uso abarca desde el campo clínico hasta estudios fisiológicos, pasando por numerosas aplicaciones prácticas de diagnóstico en finca. Objetivo: Hacer una revisión de los principios básicos de la ultrasonografía y con base en resultados de investigación, señalar la importancia de su potencial uso dentro del contexto productivo de la empresa ganadera. Métodos: Se describen conceptos anatómicos y fisiológicos del tracto reproductivo de la hembra bovina así como también sobre el uso de la ecografía en el seguimiento de la dinámica ovárica a través de mediciones del diámetro folicular y volumen del cuerpo lúteo durante el ciclo estral, relacionando estas variables con la obtención exitosa de una preñez diagnosticada igualmente por medio de ultrasonido. Resultados: Se presentan algunos referentes de investigación, así como resultados propios, que mostraron una relación positiva entre el diámetro del folículo ovulatorio, el volumen del cuerpo lúteo generado luego de la ovulación, y el diagnostico positivo de preñez. Conclusión: Nuestros datos, y la literatura relacionada, demuestran que la ultrasonografía es una herramienta útil para la evaluación, diagnóstico y toma de decisiones respecto a los eventos reproductivos de la hembra bovina, con potencial para ser usada en conjunto con la aplicación de biotecnologías reproductivas como la inseminación artificial y transferencia de embriones con el fin de optimizar su eficiencia.

Palabras clave: Ultrasonografía Transrectal, Reproducción Bovina, Dinámica Folicular, Diagnóstico de Gestación,

\section{ABSTRACT}

Background: Ultrasound has become a very useful tool in animal reproduction since implemented during the 1980's. Different applications go from clinical to physiological studies, along with a number of practical 
on-farm uses. Objective: This paper reviews the basic principles of transrectal ultrasonography and highlights the importance of its use within the productive context of cattle operations. Methods: Anatomical and physiological concepts of the cow's reproductive tract are described, along with concepts on the use of ultrasound in monitoring ovarian dynamics through follicular diameter measurements and corpus luteum volume during the estrous cycle, and this variables are related to pregnancy success also diagnosed by ultrasound. Results: Research references, as well as our own results are presented, showing a positive relationship between the diameter of the ovulatory follicle, the volume of the corpus luteum generated after ovulation, and the positive pregnancy diagnose. Conclusion: Our data, as well as the referenced literature, demonstrate that ultrasound is a useful tool, with potential for being used in evaluation, diagnosis and decisionmaking based on the cow reproductive events, along with the current reproductive biotechnologies as insemination and embryo transfer in order to optimize their efficiency.

Keywords : Trans-rectal Ultrasound, Bovine Reproduction, Follicular Dynamics, Pregnancy Diagnose.

\section{INTRODUCCIÓN}

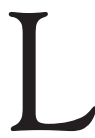

a ganadería es un sector fundamental para el crecimiento y desarrollo del País. Colombia cuenta con un censo bovino de 23,5 millones de cabezas, de las cuales 471.500 se encuentran en Norte de Santander y a su vez 192.087 son hembras en edad reproductiva. El hato ganadero aporta $1.6 \%$ al PIB nacional [1].

Uno delos pilares fundamentales de la industria ganadera bovina lo constituye el componente reproductivo, cuyo principal objetivo es obtener una cría al año para mantener una buena tasa de producción a través del tiempo. En otras palabras, no importa cuánto se mejoren todos los factores productivos (nutrición, genética, administración, sanidad), si la eficiencia reproductiva es baja, la productividad final tiende a decrecer. En concordancia con esta necesidad, desde hace más de 30 años, la ecografía ha sido valorada como una herramienta de aplicación práctica en la reproducción y diagnóstico médico [2], en diferentes especies (bovinos, equinos, ovinos).
La ultrasonografía en tiempo real es una valiosa técnica ampliamente utilizada durante los últimos años para estudiar estructuras anatómicas y funcionales del aparato reproductivo de los bovinos [3]. Diversos autores han empleado esta técnica [4, 5, 6], ya que es un método no invasivo que no solo permite el diagnóstico temprano de la gestación [7], sino que además permite realizar la evaluación del útero, cérvix, ovarios y sus cambios morfológicos, diagnóstico embrionario y fetal, sexaje del feto, seguimiento de los diferentes eventos fisiológicos presentes en los bovinos y detección y estudio de posibles cambios patológicos [8], de manera más exacta y objetiva en comparación a la técnica de palpación rectal.

El objetivo del presente artículo es presentar algunos resultados y perspectivas de investigación tendientes al incremento de la productividad, a través del mejoramiento de la eficiencia reproductiva, mediante el uso de la ultrasonografía transrectal. 


\section{PRINCIPIO DE LA ULTRASONOGRAFÍA.}

La ecografía utiliza ondas sonoras de alta frecuencia para obtener imágenes de tejidos blandos y órganos internos [8], que producen alternativamente los fenómenos de compresión y rarefacción, estas ondas son reflejadas de vuelta hacia un transductor o sonda, compuesto por un cristal piezoeléctrico (al aplicar un campo eléctrico producen sonido) recubierto en ambas caras por electrodos que al ser expuestos a una señal eléctrica, hacen que el cristal se expanda y contraiga con la misma relación que la frecuencia aplicada $(3,5,5,0,6,5$ y $7,5 \mathrm{MHz})$ [9] y posteriormente son enviadas al ecógrafo donde son analizadas y convertidas en una imagen en escala de grises, que se extiende desde el negro al blanco. Las estructuras contenidas por líquidos (folículos, saco vitelino, algunos órganos), que se visualizan en color negro, no reflejan las ondas sonoras, por lo que son denominadas anecogénicas o no ecogénicas, cuando la mayor parte del sonido se refleja hacia el transductor y se refleja una imagen de color blanco, se habla de imagen ecogénica, como en el caso de huesos y aire. Los tejidos blandos pueden aparecer en distintas clases de grises así, hiperecogénico define a los tejidos que reflejan más sonido que el tejido circundante (huesos del feto) e hipoecogénico que describe la idea contraria (cuerpo lúteo).

\section{ASPECTOS ANATÓMICOS DEL TRACTO GENITAL DE LA HEMBRA BOVINA}

El aparato reproductor de la hembra bovina posee un útero bicorneo, constituido por los cuernos, el cuerpo y el cuello, que conecta los oviductos y vagina [10]. El ovario es la gónada femenina compuesta por estructuras como el folículo y el cuerpo lúteo, a su vez es la encargada de la producción y secreción de hormonas sexuales (Estrógenos y Progesterona) y cuya unidad funcional es el folículo que contiene los oocitos que pueden llegar a la ovulación, ser fertilizados y formar un embrión [11]. Los folículos contienen líquido y son identificados como estructuras anecogénicas por ultrasonido, por el contrario el cuerpo lúteo está conformado por tejido sólido y su imagen ecográfica se refleja de manera hipoecogénica.

La emergencia de una onda folicular ha sido definida como el crecimiento de folículos de más de $4 \mathrm{~mm}$ de diámetro y es caracterizada por la manera retrospectiva que el folículo dominante tiende a ser seleccionado de la cohorte de folículos en crecimiento, al alcanzar un diámetro aproximado de $8,5 \mathrm{~mm}$ (desviación folicular), el folículo dominante continua su crecimiento mientras que los folículos subordinados reducen su crecimiento y disminuyen su tamaño (atresia), después del pico preovulatorio de $\mathrm{LH}$ surge la ovulación y posteriormente la formación del cuerpo lúteo es iniciado por una serie de cambios morfológicos y bioquímicos en las células de la teca interna (TI) y de la granulosa (CG) del folículo preovulatorio [12].

\section{ECOGRAFÍA DE LA DINÁMICA OVARICA}

La implementación de la ultrasonografía transrectal en bovinos ha permitido dilucidar los mecanismos ováricos que suceden durante la reactivación ovárica posparto, el ciclo estral (Describir ondas de crecimiento y atresia folicular, dinámica del cuerpo lúteo, ovulación), estimación del número de folículos en el caso de la transferencia de embriones y los tratamientos de superovulación [13], identificación quistes foliculares o luteales, que antes no eran conocidos.

Gracias a esta técnica y al hallazgo de que el crecimiento de folículos bovinos ocurre en un patrón denominado ondas de crecimiento folicular [14], ha sido posible el diseño e implementación de protocolos de sincronización de ovulación para programas de Inseminación artificial (IA) $y$ sus modificaciones $[15,16,17,18,19,20]$, permitiendo un incremento valioso en la productividad y la calidad genética gracias a la masificación del uso de la IA.
Enero - Junio 2014

ISSN 0122-820X

PP: 99-106 
No. 1

Enero - Junio 2014 ISSN 0122-820X

PP: 99-106
Los folículos pueden ser visualizados, cuantificados y supervisados de forma secuencial a partir de los 2-3 mm [21], se muestran a través de imágenes no ecogénicas de color negro en forma redondeada o en estructuras irregulares debido a la compresión de los folículos adyacentes, al CL y a la compresión de los folículos por el estroma ovárico (Fig.1). Los folículos preovulatorios se muestran como estructuras redondeadas anecogénicas de 15-17 m, tamaño con el cual se espera la ovulación en la vaca [2]. La ovulación puede ser asumida con la desaparición del folículo preovulatorio y la posterior aparición de células luteales [13] el cuerpo lúteo se muestra como una imagen hipoecogenica algo oscura y redondeada, alrededor de los 2-3 días posteriores a la ovulación.

Figura1. Imágenes ecográficas de la dinámica ovárica. cohorte de folículos (1); fase de dominancia en la dinámica ovárica (2); folículo preovulatorio (3) y cuerpo lúteo establecido (4)

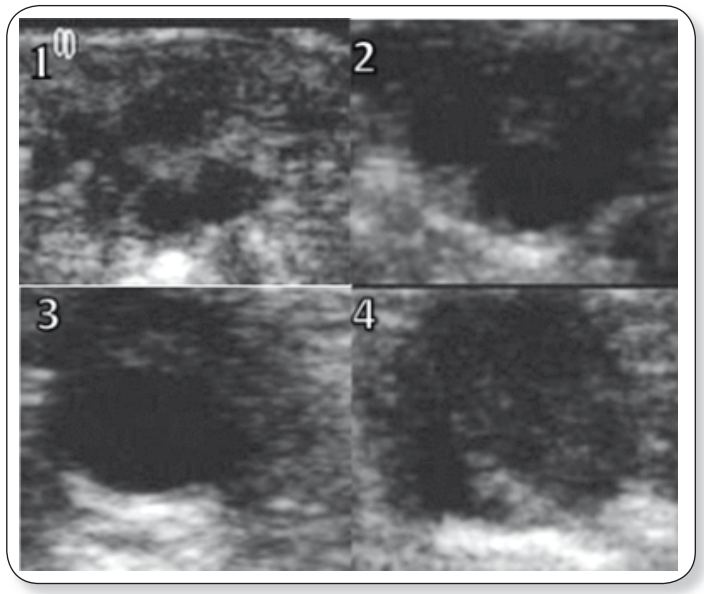

Fuente: Adaptado de Gutiérrez, 2012 [23].

\section{DIAGNÓSTICO TEMPRANO DE LA GESTACIÓN}

El diagnóstico de gestación mediante la ecografía, se basa en la presencia de líquido uterino intraluminal [13], el cual se muestra a través de imágenes no ecogénicas, como en el caso de los folículos, es posible observar el embrión como una imagen ecogénica a partir del 27 posterior a la fecundación con una especificidad del $86 \%[9,23]$, un diagnostico antes de esta fecha o por la presencia de líquido en el útero no es confiable, ya que puede confundirse con alguna patología como el piométra.

Figura 2. Diagnóstico de gestación por ecografía. 30 días de preñez (1); 37 dias de preñez (2); 48 días de preñez (3) y 55 días de preñez (4).

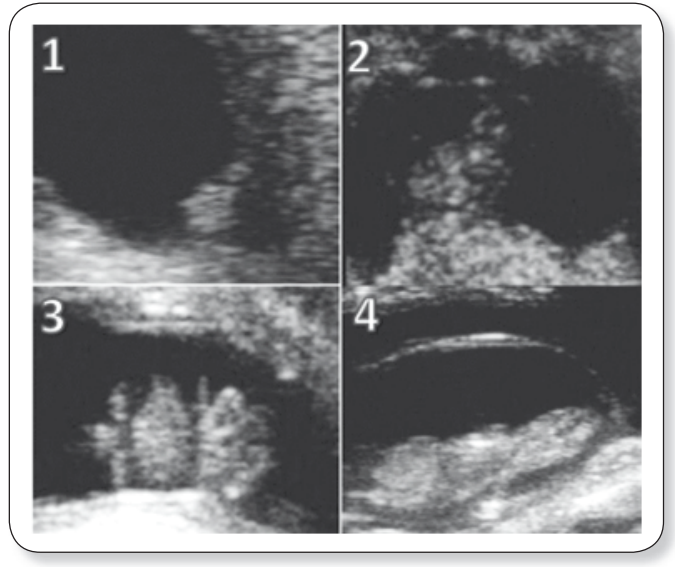

Fuente: Adaptado de Gutiérrez, 2012 [23]

Una de las aplicaciones prácticas más empleadas de la ultrasonografía transrectal, es la evaluación del establecimiento o no de una preñez y su estado, al identificar a tiempo que una vaca servida no se encuentra gestante, es posible reducir el intervalo entre servicios, el aumento en la tasa de servicios y por ende los días abiertos [13]. Por otro lado el diagnóstico temprano de la gestación es de gran utilidad en los programas de Inseminación artificial, ya que la selección de las hembras es más precisa, evitando que ingresen vacas con preñez temprana (menor a 45 días), que al ser sometidas a tratamientos hormonales como en el caso de la Prostaglandina, puedan presentar un aborto. Adicionalmente el útero también puede evaluarse para las condiciones patológicas, como por ejemplo, metritis, piometra, feto macerado o momificado [7].

\section{AVANCES E INVESTIGACIONES EN EL USO DE LA ULTRASONOGRAFÍA}

En Colombia diversos autores han empleado la técnica de ultrasonografía transrectal en hembras tipo carne y tipo leche, como herramienta de diagnóstico reproductivo tales como: Describir la dinámica folicular durante el anestro posparto y el primer ciclo estral con presencia o ausencia del ternero 
en vacas Brahman [24], caracterizar el comportamiento folicular ovárico durante el ciclo estral de hembras Blanco Orejinegro (BON) [25], evaluar el efecto del diámetro del folículo ovulatorio, tamaño del cuerpo lúteo y perfiles de progesterona sobre la tasa de preñez en novillas Holstein Friesian, receptoras de embriones bovinos [6], evaluar el desarrollo del cuerpo lúteo y tasa de preñez en hembras raza Holstein, receptoras de embriones bovinos sometidas a la aplicación de eCG [26]

El Grupo de Investigación en Ciencias Agronómicas y Pecuarias (GICAP-UFPS), en su Línea de Investigación en Reproducción Animal, ha mantenido esfuerzos para que tanto la Institución como Norte de Santander estén a la vanguardia en el uso y aprovechamiento de los avances tecnológicos que han revolucionado el conocimiento de la biología reproductiva, como muestra Gutiérrez [23], mediante el uso de la ultrasonografía transrectal, estableció la relación entre el diámetro del folículo ovulatorio y el diagnostico positivo de gestación en vacas lactantes, mantenidas en un sistema de monta natural, determinando las probabilidades de preñez para diferentes diámetros foliculares, donde el incremento en la tasa de preñez estuvo asociado al mayor diámetro del folículo ovulatorio, el cual fue en promedio de 16,8 \pm 1,67 mm, (Tabla I),

Tabla I. Diámetro del folículo ovulatorio (dfo) y volumen del cuerpo lúteo (vcl) (vacas no preñadas (n) vs preñadas (pñ))

\begin{tabular}{ccccc}
\hline Variable & Preñez & N & Promedio \pm DE & P \\
\hline DFO $(\mathrm{mm})$ & N & 6 & $15.0 \pm 1.67$ & $0.0295^{*}$ \\
& Pñ & 12 & $16.8 \pm 1.47$ & \\
VCL & N & 6 & $545.5 \pm 196.8$ & $0.0001^{* *}$ \\
& Pñ & 12 & $2350.6 \pm 347.5$ & \\
$\left(\mathrm{~mm}^{3}\right)$ & & & & \\
\hline
\end{tabular}

*Diferencias significativas $(\mathrm{p}<0.05)$.

**Diferencias altamente significativas $(p<0.01)$.

En cuanto a la reactivación ovárica posparto se logró evidenciar el desarrollo de ondas foliculares durante el periodo posparto, el ciclo estral y la preñez temprana, que incluyen el crecimiento y atresia folicular en periodos de celo a ovulación, así como también fue posible la diferenciación de fases como formación, crecimiento y regresión o lisis del cuerpo lúteo.

En la figura 3 se presentan gráficamente los perfiles de dinámica folicular (diámetro de los folículos presentes a través del tiempo) y luteal (diámetro del CL) obtenidas a partir de los registros ecográficos de la evaluación periódica de la dinámica ovárica, el establecimiento y mantenimiento del cuerpo lúteo, diagnóstico y seguimiento de la gestación durante todo el periodo de investigación.

Figura 3. Dinámica ovárica posparto de dos hembras bovinas

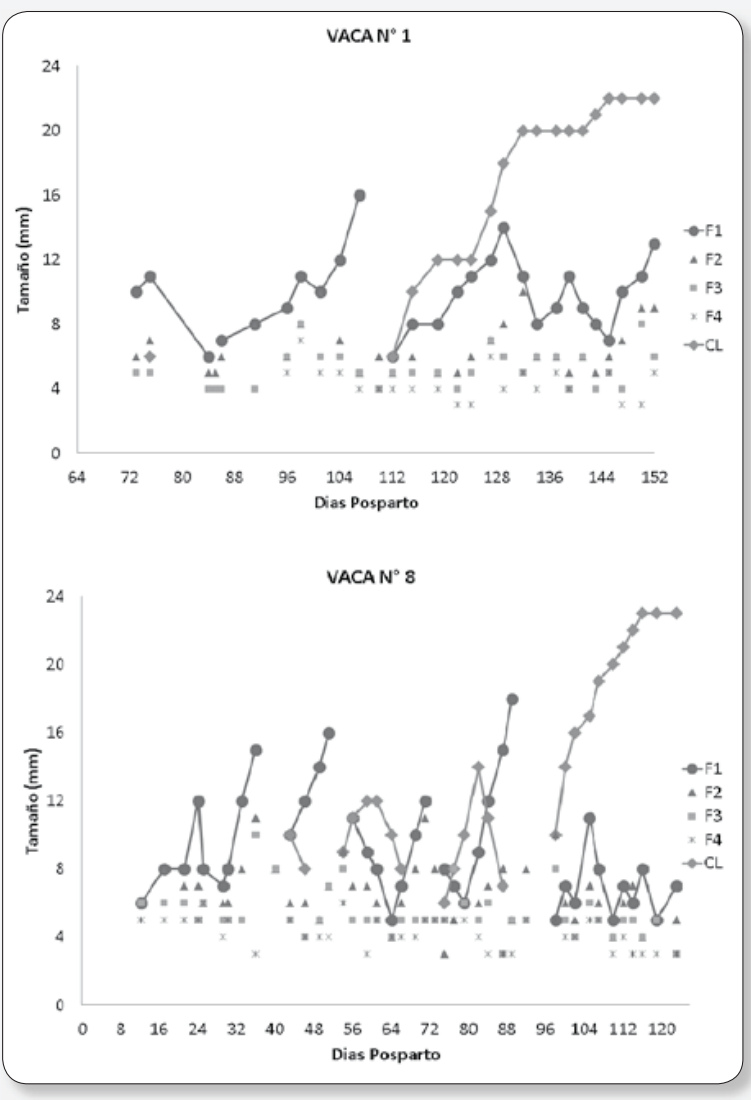

Enero - Junio 2014 ISSN 0122-820X

PP: 99-106

Fuente: Gutiérrez, 2012 [23]

Adicionalmente mediante la aplicación de esta técnica de ultrasonografía se pudo evidenciar una hembra que desarrollo un quiste folicular (Fig. 4.) 
No. 1

Enero - Junio 2014

ISSN 0122-820X

PP: 99-106
Figura 4. Quiste folicular

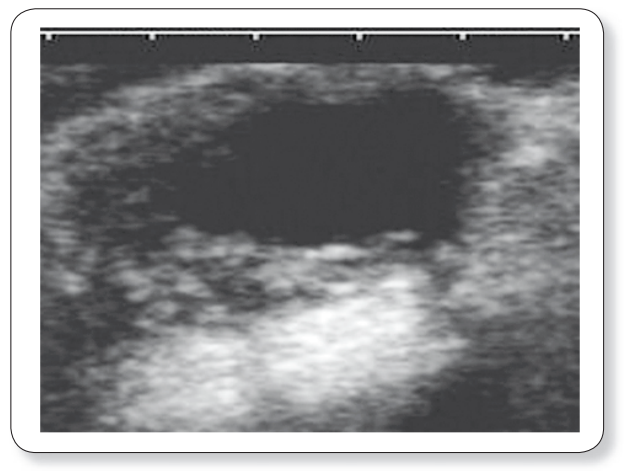

Fuente: Gutiérrez, 2012 [23]

\section{INVESTIGACIONES ADICIONALES Y EXTENSION A GANADEROS.}

En un proyecto de Inseminación Artificial a Tiempo Fijo (IATF), desarrollado en el marco de una práctica estudiantil de la UFPS, se utilizó la ultrasonografía transrectal como herramienta de apoyo y diagnóstico [27], realizando la confirmación de preñez a un grupo de hembras que habían sido sometidas a un protocolo de IATF, donde fue posible diagnosticar una hembra en estadodegestación (Fig. 5.) de 10 que habían sido tratadas con un protocolo a base de progesterona, estrógenos y prostaglandina.

Figura 5. Gestación de 45 días

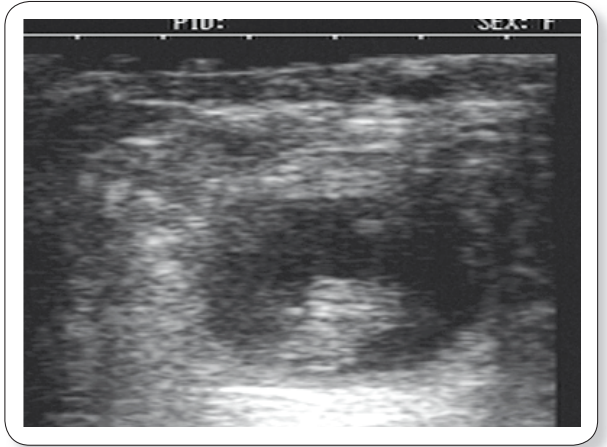

Fuente: Gutiérrez, 2012 [23]

Trabajos adicionales de extensión y formación académica se han desarrollado en el marco de la cátedra de reproducción animal de la UFPS, en la Finca La Esperanza, Norte de Santander, se logró identificar mediante un examen ecográfico previo a la aplicación de una dosis de prostaglandina, que 5 de 10 hembras que habían sido seleccionadas para un programa de IATF, presentaban una gestación temprana
(Fig.6.), la cual no fue diagnosticada mediante la palpación rectal al momento de la selección, evitando la inducción a un posible aborto, de igual forma fue posible determinar los diámetros de los folículos preovulatorios y posteriormente realizar la confirmación de preñez de las vacas inseminadas.

Figura 6. Imágenes ecograficas de preñeces tempranas

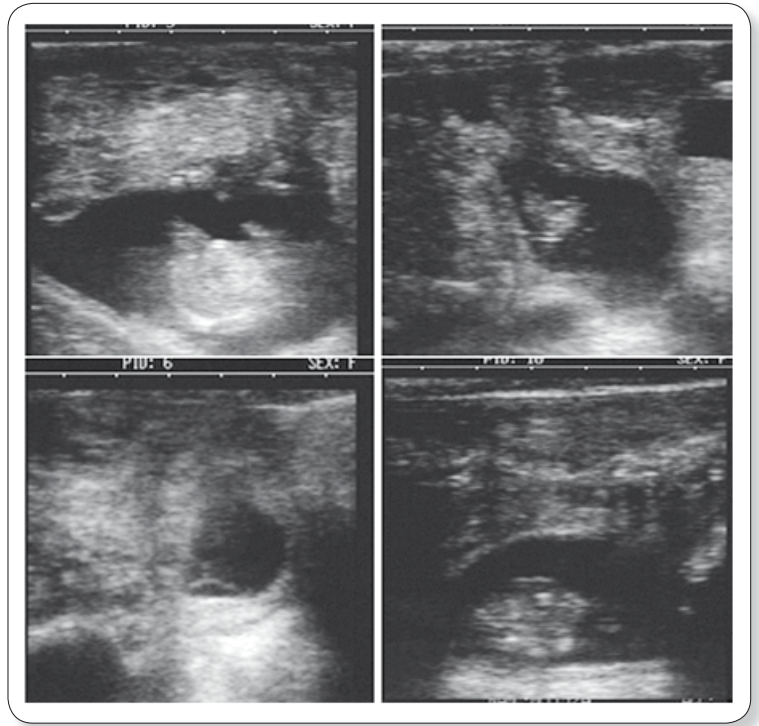

Fuente: Baéz, datos sin publicar

Estos resultados contrastan con los obtenidos por Galán [27], y demuestran como el uso de la ultrasonografía durante la fase de selección para iniciar un protocolo de IATF, es de gran ayuda a la hora de tomar decisiones en cuanto al manejo de las hembras, o para este caso en particular, el diagnóstico de gestación temprana, la cual en muchas ocasiones no puede ser detectada mediante la palpación rectal, siendo de gran utilidad para evitar posteriores inconvenientes como abortos, malformaciones, etc.

\section{CONCLUSIONES}

La ultrasonografía transrectal es una valiosa herramienta para incrementar la eficiencia reproductiva de la hembra bovina, a través del seguimiento y evaluación de variables como el diámetro del folículo preovulatorio, volumen luteal, y diagnóstico de gestación, que permiten predecir respuestas y evaluar programas de monta natural, inseminación 
artificial o transferencia de embriones, con potencial aplicabilidad en las áreas productiva, investigativa y académica.

El grupo GICAP-UFPS en su línea de investigación en reproducción animal, proyecta continuar las investigaciones en este campo, mediante el desarrollo de trabajos de grado en sus modalidades: investigación o pasantía y extensión a ganaderos de la región, con el fin de identificar posibles puntos críticos que afecten el desempeño reproductivo de las hembras y de esta manera formular estrategias que mejoren los parámetros, así como también consolidar redes de apoyo con centros de investigación o entidades del sector público y/o privado.

\section{AGRADECIMIENTOS}

Los trabajos de ultrasonografía referenciados en el presente documento [23, 27] fueron posibles gracias a la adquisición del equipo de ultrasonido mediante financiación FINUUPFS, Contrato 011-2011.

Agradecimientos a Productos de la Hacienda Parra y su representante, Alfonso Enrique Parra Montoya, por permitir la realización de una de las investigaciones [23], al Tecnólogo Agropecuario Luis Carlos Galán, quien desarrollo trabajos de inseminación [27] en la Finca Las Vegas (Danilo Castro), con semen donado por Leonardo Hernández (SENA), y a los estudiantes que participaron en los diferentes proyectos, incluido el desarrollado en la Finca La Rinconada, propiedad de José Trinidad Páez.

\section{BIBLIOGRAFÍA}

[1] FEDEGAN - FNG, Subgerencia de Salud y Bienestar Animal. Censo Nacional de Predios y Bovinos, 2011.

[2] DesCôteaux L, Gnemmi G and Colloton J. Ultrasonography of the Bovine Female Genital Tract. Vet Clin Food Anim 2009; 25: 733-752

[3] Perea F. Manual de Ganadería Doble Pro- pósito: Ecografía reproductiva. Trujillo: Núcleo Universitario Rafael Rangel, Universidad de Los Andes; 2005.

[4] Motta PA, Ramos N, González CM, Castro EC. Dinámica folicular en la vida reproductiva de la hembra bovina. Vet zootec 2011; 5 (2): 88-99.

[5] Rahman MS, Shohag AS, Kamal MM, Bari FY, Shamsuddin M. Preovulatory folicular and subsequent luteal size influence pregnancy success in water buffaloes. J Reprod Dev 2012; 58: 219-222.

[6] Duica, A., Efecto del diámetro del folículo ovulatorio, tamaño del cuerpo lúteo y perfiles de progesterona sobre la tasa de preñez en la hembra receptora de embriones bovinos, Tesis de Maestría. Bogotá: Facultad de Medicina Veterinaria y de Zootecnia, Universidad Nacional de Colombia, 2010. p.190

[7] Quintela LA, Barrio M, Peña AI, Becerra, JJ, Cainzos J, Herrado PG and Díaz C. Use of Ultrasound in the Reproductive Management of Dairy Cattle. J. Reprod Dom Anim 2012; 47 (Suppl. 3): 34-44.

[8] Pierson RA, Kastelic JP and Ginther OJ. Basic principles and techniques for transrectal ultrasonography in cattle and horses. Theriogenology 1988; 29 (1)

[9] Quintela LA, Díaz C, Herradón PG, Peña AI, Becerra JJ. Ecografía y reproducción en la vaca. Ed. Servicio de publicaciones e intercambio científico, Universidad Santiago de Compostela, Spain, 2006. 92p.

[10] Senger, P.L. Pathways to Pregnancy and Parturition, second edition, Washington, 2003. p. 373

[11] Findlay JK et al. Ovarian physiology: follicle development, oocyte and hormone relationships, Anim. Reprod. 2009; v.6, n.1, p.16-19. ISSN 0122-820X

PP: 99-106
[12] Wiltbank MC et al, Managing the dominant follicle in lactating dairy cows., Advances in Bovine Reproduction and Embryo Technology., Theriogenology 76 (2011) 1568-1582 
No. 1

Enero - Junio 2014 ISSN 0122-820X

PP: 99-106
[13] Fricke PM. Scanning the FutureUltrasonography as a Reproductive Management Tool for Dairy Cattle. J. Dairy Sci. 2002; 85:1918-1926

[14] Wiltbank MC, Pursley JR, Fricke PM et al. Development of AI and ET programs that do not require detection of estrus using recent information on follicular growth. Proceedings of the XV annual convention AETA, memorias.1996. 62p.

[15] Pursley JR, Mee MO and Wiltbank MC. Synchronization of ovulation in dairy cows usingPGF $2 \alpha$ and GnRH. Theriogenology 1995; 44:915-923.

[16] Pursley JR, Kosorok MR and Wiltbank MC. Reproductive management of lactating dairy cows using synchronization of ovulation. J. Dairy Sci. 1997; 80:301-306

[17] Vasconcelos JLM, Silcox RW, Rosa GJ, PursleyJR, and Wiltbank MC. Synchronization rate, size of the ovulatory follicle, and pregnancy rate after synchronization of ovulation beginning on different days of the estrous cycle in lactating dairy cows. Theriogenology 1999; 52:1067-1078

[18] Moreira F, De la Sota RL, Diaz T, and Thatcher WW. Effect of day of the estrous cycle at the initiation of a timed artificial insemination protocol on reproductive responses in dairy heifers. J. Anim. Sci. 2000a. 78:1568-1576.

[19] Moreira F, Risco CA, Pires MFA, Ambrose JD, Drost M, and Thatcher WW. Use of bovine somatotropin in lactating dairy cows receiving timed artificial insemination. J. Dairy Sci. 2000b; 83:1237-1247.

[20] Gumen A, Keskin A, Yilmazbas-Mecitoglu G, Karakaya E, Alkan A, Okut H, Wiltbank MC. Effect of presynchronization strategy before Ovsynch on fertility at first service in lactating dairy cows. Theriogenology. 2012 Nov;78(8):1830-8

[21] Pierson RA and Ginther OJ. Ultrasonic imaging of the ovaries and uterus in cattle. Theriogenology, 1988; 29 (1): 21-37
[22] Tamayo, M., La ecografía como medio diagnóstico y evaluación de los procesos reproductivos en el bovino. Departamento de Clínica, Facultad de Medicina Veterinaria, Universidad Agraria de La Habana. Disponible en: http://www.google.com/ url?sa $=\mathrm{D} \& \mathrm{q}=\mathrm{http}: / \mathrm{www} \cdot$ produccionanimal. com.ar/infrmacion_tecnica/ecografia_ultrsonido/36 ecografia_reproduccion.pdf

[23] Gutiérrez, DE. Relación entre el folículo ovulatorio y la tasa de preñez en vacas lactantes. Tesis de Grado. Cúcuta. Facultad de Ciencias Agrarias y del Ambiente. Universidad Francisco de Paula Santander. 2012. 57p

[24] Henao G, Olivera M, Maldonado JG. Follicular dynamics during postpartum anestrus and the first estrous cycle in suckled or non-suckled Brahman (Bos indicus) cows. Anim Reprod Sci 2000; 63: 127-136.

[25] Henao D, Carrillo L, Olivera M. Comportamiento durante el calor y dinámica folicular interestral en vacas BON (Blanco Orejinegro), Rev Col CiencPec 2004; Vol. 17:1

[26] Tovio N. Efectos de la aplicación de eCG (Día 5 u 8) sobre el desarrollo del cuerpo lúteo, nivel de progesterona y tasa de preñez en hembras receptoras de embriones bovinos. Tesis de Maestría. Bogotá: Facultad de Medicina Veterinaria y de Zootecnia, Universidad Nacional de Colombia, 2011. p.157

[27] Galán LC. Apoyo Tecnológico En El Manejo Reproductivo De La Finca Las Vegas. (Puerto Nuevo, Corregimiento Buena Esperanza Municipio De San José De Cúcuta (Norte De Santander)), Trabajo de Grado. Universidad Francisco de Paula Santander. 2011.67p 